\title{
THE IDEMPOTENT-SEPARATING CONGRUENCES ON A REGULAR 0-BISIMPLE SEMIGROUP
}

\author{
by W. D. MUNN
}

(Received 1st September 1966)

A congruence $\rho$ on a semigroup is said to be idempotent-separating if each $\rho$-class contains at most one idempotent. For any idempotent $e$ of a semigroup $S$ the set $e S e$ is a subsemigroup of $S$ with identity $e$ and group of units $H_{e}$, the maximal subgroup of $S$ containing $e$. The purpose of the present note is to show that if $S$ is a regular 0-bisimple semigroup and $e$ is a non-zero idempotent of $S$ then there is a one-to-one correspondence between the idempotentseparating congruences on $S$ and the subgroups $N$ of $H_{e}$ with the property that $a N \subseteq N a$ for all right units $a$ of $e S e$ and $N b \subseteq b N$ for all left units $b$ of $e S e$. Some special cases of this result are discussed and, in the final section, an application is made to the principal factors of the full transformation semigroup $\mathscr{T}_{X}$ on a set $X$.

1. The notation of (1) will be used throughout. In particular, if $\rho$ is an equivalence on a set $X$ then $x \rho$ denotes the $\rho$-class containing the element $x$ of $X$. As in (1), an exception is made in the case of Green's equivalences $\mathscr{R}, \mathscr{L}$ and $\mathscr{H}$ on a semigroup $S$ : the corresponding classes containing the element $a$ of $S$ are denoted by $R_{a}, L_{a}$ and $H_{a}$.

Let $S$ contain an idempotent $e$. Then $e S e$ is a subsemigroup of $S$ and we write

$$
S_{e}=e S e, P_{e}=R_{e} \cap S_{e}, Q_{e}=L_{e} \cap S_{e} .
$$

Since $e$ is a left identity for $R_{e}$ and a right identity for $L_{e}$ we see that

$$
P_{e}=\left\{x \in R_{e}: x e=x\right\}, Q_{e}=\left\{x \in L_{e}: e x=x\right\} .
$$

In the first two lemmas we establish some basic properties of these sets.

Lemma 1. Let e and $f$ be $\mathscr{D}$-equivalent idempotents of a semigroup $S$. Then $S_{e} \cong S_{f}$.

Proof. Since $(e, f) \in \mathscr{D}$ there exists an element $a$ in $R_{e} \cap L_{f}$. By $((\mathbf{1})$, Theorem 2.18), there is a unique element $a^{\prime}$ in $R_{f} \cap L_{e}$ such that $a a^{\prime}=e$ and $a^{\prime} a=f$. Let $x \in S_{e}$. Then

$$
a^{\prime} x a=a^{\prime} \text { exea }=f a^{\prime} x a f \in S_{f} \text {. }
$$

Similarly, if $y \in S_{f}$ then aya' $\in S_{e}$. Now let $\theta: S_{e} \rightarrow S_{f}$ and $\phi: S_{f} \rightarrow S_{e}$ be defined by $x \theta=a^{\prime} x a\left(x \in S_{e}\right)$ and $y \phi=a y a^{\prime}\left(y \in S_{f}\right)$. Then $x \theta \phi=e x e=x$ for all $x \in S_{e}$. Similarly, $y \phi \theta=y$ for all $y \in S_{f}$. Hence $\theta$ and $\phi$ are mutually E.M.S. $-Q$ 
inverse bijections. Finally, $a^{\prime} x y a=\left(a^{\prime} x a\right)\left(a^{\prime} y a\right)$ for all $x, y \in S_{e}$ and so $\theta$ is an isomorphism.

In particular, if $S$ is a regular 0-bisimple semigroup then $S_{e} \cong S_{f}$ for any non-zero idempotents $e, f$. In this case it can also be shown that $S_{e}$ is 0 -bisimple.

Let $T$ be a semigroup with an identity $e$. By a right unit of $T$ we mean an element $a$ of $T$ such that $a x=e$ for some $x$ in $T$. The set of all right units of $T$ is readily seen to be a right cancellative subsemigroup of $T$ (called the right unit subsemigroup of $T$ ). Left units are defined in a similar way; the set of all such elements is a left cancellative subsemigroup of $T$ (the left unit subsemigroup of $T$ ). The elements of $T$ that are both right units and left units are called units.

Lemma 2. Let $e$ be an idempotent of a semigroup $S$. Then $P_{e}$ is the right unit subsemigroup of $S_{e}$ and $Q_{e}$ is the left unit subsemigroup of $S_{e}$. The group of units of $S_{e}$ is $H_{e}$.

Proof. Let $a \in P_{\mathrm{e}}$. Then there exists $x \in S^{1}$ such that $a x=e$. Write $y=e x e$. Since $a e=a$ we have that $a y=a x e=e^{2}=e$. Hence $a$ is a right unit of $S_{e}$. Conversely, let $a$ be a right unit of $S_{e}$. Then there exists $y \in S_{e}$ such that $a y=e$. Also $e a=a$ and so $a \in R_{e} \cap S_{e}=P_{e}$. Thus $P_{e}$ is the right unit subsemigroup of $S_{e}$. Similarly, $Q_{e}$ is the left unit subsemigroup of $S_{e}$.

Finally, $P_{e} \cap Q_{e}=R_{e} \cap L_{e} \cap S_{e}=H_{e}$.

2. By a left normal divisor of $P_{e}$ we shall mean a subgroup $N$ of $H_{e}$ such that $a N \subseteq N a$ for all $a \in P_{e}$. Since $H_{e} \subseteq P_{e}$ it is clear that, in particular, a left normal divisor of $P_{e}$ is a normal subgroup of $H_{e}$. Similarly, by a right normal divisor of $Q_{e}$ we mean a subgroup $N$ of $H_{e}$ such that $N b \subseteq b N$ for all $b \in Q_{e}$. This terminology is due to Rees (5). In the next lemma we establish a connection between such subgroups of $H_{e}$ and the congruences on $S$ contained in $\mathscr{H}$.

Lemma 3. Let e be an idempotent of a semigroup $S$. Let $\rho$ be any congruence on $S$ contained in $\mathscr{H}$ and let $N=e \rho$. Then $N$ is a left normal divisor of $P_{e}$ and a right normal divisor of $Q_{e}$.

Proof. Since $\rho \cap\left(H_{e} \times H_{e}\right)$ is a congruence on $H_{e}$ and $\rho \subseteq \mathscr{H}$ it follows that $N$ is a normal subgroup of $H_{e}$. Let $a \in P_{e}$ and let $b \in a \rho$. Then since $\rho \subseteq \mathscr{H}$ there exists $x^{\prime} \in S^{1}$ such that $x^{\prime} a=b$. Write $x=x^{\prime} e$; then $x a=x^{\prime} e a=x^{\prime} a=b$ and $x e=x$. Also since $a \rho \subseteq H_{a} \subseteq R_{e}$ there exists $y \in S^{1}$ such that $b y=e$. Now $(x b y, x a y) \in \rho$ since $\rho$ is a congruence on $S$; that is, $(x, e) \in \rho$. Thus $x \in N$. Hence, since $b=x a$, we have that $a \rho \subseteq N a$.

Next let $z \in N$. Then $(a z, a e) \in \rho$. But $a e=a$; hence $a N \subseteq a \rho$. Combining these results we see that $a N \subseteq N a$.

In the same way we can show that $N b \subseteq b N$ for all $b \in Q_{e}$.

Corollary. If $\mathscr{H}$ is a congruence on $S$ then $H_{e}$ is a left normal divisor of $P_{e}$ and a right normal divisor of $Q_{\boldsymbol{e}}$.

Does every subgroup of $H_{e}$ that is both a left normal divisor of $P_{e}$ and a right normal divisor of $Q_{e}$ arise, as in Lemma 3 , from a congruence contained in $\mathscr{H}$ ? This is answered for regular 0-bisimple semigroups in Lemma 6 . As 
a first step we establish

Lemma 4. Let e be an idempotent of a semigroup $S$.

(i) Let $a, b \in R_{\mathbf{e}}$. Then

$$
(a, b) \in \mathscr{H} \Leftrightarrow x a=b \text { for some } x \in H_{e} .
$$

(ii) Let $N$ be a left normal divisor of $P_{e}$. Define a relation $\rho_{R}$ on $R_{e}$ by the rule that

$$
(a, b) \in \rho_{R} \Leftrightarrow x a=b \text { for some } x \in N .
$$

Then $\rho_{R}$ is an equivalence on $R_{e}$ contained in $\mathscr{H}$. Further, if $(a, b) \in \rho_{R}$ then $(c a, c b) \in \rho_{R}$ for all $c \in P_{e}$.

Proof. (i) Since $(e, a) \in \mathscr{R}$ we have $e a=a$ and so $H_{e} a=H_{a}((1)$, Lemma 2.2). This gives the required result.

(ii) That $\rho_{R}$ is an equivalence on $R_{e}$ follows from the fact that $N$ is a group whose identity $e$ is a left identity for $R_{e}$. From (i) we see that $\rho_{R} \subseteq \mathscr{H}$.

Let $(a, b) \in \rho_{R}$ and let $c \in P_{e}$. Since $a \in R_{e}$ there exists $z \in S^{1}$ such that $a z=e$. Hence $c a z=c e=c$ and so $(c a, c) \in \mathscr{R}$; that is, $c a \in R_{e}$. Similarly, $c b \in R_{e}$. Now $x a=b$ for some $x \in N$ and $c N \subseteq N c$, by hypothesis. Hence

$$
c b=c x a=y c a
$$

for some $y \in N$, which shows that $(c a, c b) \in \rho_{R}$.

Dually, for any right normal divisor $N$ of $Q_{e}$ we define a relation $\rho_{L}$ on $L_{e}$ by the rule that

$$
(a, b) \in \rho_{L} \Leftrightarrow a x=b \text { for some } x \in N .
$$

Then $\rho_{L}$ is an equivalence on $L_{e}$ contained in $\mathscr{H}$ and if $(a, b) \in \rho_{L}$ then $(a c, b c) \in \rho_{L}$ for all $c \in Q_{e^{*}}$

3. In this section we restrict our attention to 0-bisimple semigroups. By ((1), Theorem 2.11) such a semigroup is regular if and only if it contains a nonzero idempotent.

Lemma 5. Let $S$ be a 0-bisimple semigroup and let $a$ be an arbitrary but fixed non-zero element of $S$. Let $\rho, \tau$ be congruences on $S$ contained in $\mathscr{H}$. Then

(i) $\rho=\mathscr{H}$ if and only if $a \rho=H_{a}$;

(ii) $\rho \subseteq \tau$ if and only if $a \rho \subseteq a \tau$.

Proof. (i) Let $a \rho=H_{a}$. To establish (i) we need only prove that $\mathscr{H} \subseteq \rho$. First, $H_{0}=0 \rho=0$. Now let $(b, c) \in \mathscr{H}$, where $b \neq 0, c \neq 0$. Since $S$ is 0-bisimple there exist elements $s, s^{\prime}, t, t^{\prime}$ in $S^{1}$ such that $b=s a t, a=s^{\prime} b t^{\prime}$ and the mappings

$$
x \rightarrow \operatorname{sxt}\left(x \in H_{a}\right), y \rightarrow s^{\prime} y t^{\prime}\left(y \in H_{b}\right)
$$

are mutually inverse bijections from $H_{a}$ to $H_{b}$ and from $H_{b}$ to $H_{a}$ respectively ((1), Theorem 2.3). Thus, since $(b, c) \in \mathscr{H}$, we see that $\left(s^{\prime} b t^{\prime}, s^{\prime} c t^{\prime}\right) \in \mathscr{H}$. Since $a=s^{\prime} b t^{\prime}$ and $a \rho=H_{a}$ it follows that $\left(a, s^{\prime} c t^{\prime}\right) \in \rho$. But $\rho$ is a congruence on $S$ and so $\left(s a t, s s^{\prime} c t^{\prime} t\right) \in \rho$; that is, $(b, c) \in \rho$. Thus $\mathscr{H} \subseteq \rho$. 
(ii) It is clear that if $\rho \subseteq \tau$ then $a \rho \subseteq a \tau$. Suppose, conversely, that $a \rho \subseteq a \tau$. Let $(b, c) \in \rho$, where $b \neq 0, c \neq 0$. To prove (ii) it suffices to show that $(b, c) \in \tau$. As above, since $(a, b) \in \mathscr{D}$ there exist elements $s, s^{\prime}, t, t^{\prime}$ in $S^{1}$ such that $b=s a t, a=s^{\prime} b t^{\prime}$ and the mappings $x \rightarrow s x t\left(x \in H_{a}\right), \quad y \rightarrow s^{\prime} y t^{\prime}\left(y \in H_{b}\right)$ are mutually inverse bijections from $H_{a}$ to $H_{b}$ and from $H_{b}$ to $H_{a}$ respectively. Since $\rho$ is a congruence, $\left(s^{\prime} b t^{\prime}, s^{\prime} c t^{\prime}\right) \in \rho$; that is, $\left(a, s^{\prime} c t^{\prime}\right) \in \rho$. But $a \rho \subseteq a \tau$ and so $\left(a, s^{\prime} c t^{\prime}\right) \in \tau$. Hence $\left(s a t, s s^{\prime} c t^{\prime} t\right) \in \tau$ since $\tau$ is a congruence. But $(b, c) \in \mathscr{H}$ since $\rho \subseteq \mathscr{H}$. Thus $s s^{\prime} c t^{\prime} t=c$. It follows that $(b, c) \in \tau$, as required.

In particular, from (ii) above, $\rho=\tau$ if and only if $a \rho=a \tau$ for any non-zero element $a$ of $S$; that is, a congruence contained in $\mathscr{H}$ on a 0 -bisimple semigroup is uniquely determined by any one of its non-zero classes.

We now come to the key result.

Lemma 6. Let $S$ be a regular 0-bisimple semigroup and let e be a non-zero idempotent of $S$. Let $N$ be a subgroup of $H_{e}$ that is both a left normal divisor of $P_{e}$ and a right normal divisor of $Q_{e}$. Then there exists a congruence $\rho$ on $S$ contained in $\mathscr{H}$ and such that $e \rho=N$.

Proof. We construct $\rho$ by defining $\rho \cap(H \times H)$ for each non-zero $\mathscr{H}$-class $H$ in terms of the equivalence $\rho_{R}$ on $R_{e}$ described in Lemma 4 (ii). The argument depends on several applications of the dual of ((1), Lemma 2.2) which we shall refer to below as Green's lemma.

Let $(a, b) \in \mathscr{H}$, where $a \neq 0, b \neq 0$. Since $S$ is 0 -bisimple there exists an element $s \in S^{1}$ such that $s a \in R_{e} \cap L_{a}$. Then $s b \in R_{e} \cap L_{a}$ by Green's lemma. Now let $(s a, s b) \in \rho_{R}$. We prove first that $(z a, z b) \in \rho_{R}$ for $a n y z \in S^{1}$ such that $z a \in R_{e} \cap L_{a}$. By Lemma 4 (i), since $(s a, z a) \in \mathscr{H}$, there exists $x \in H_{e}$ such that $x s a=z a$. Also since $(a, b) \in \mathscr{H}$, there exists $y \in S^{1}$ such that $a y=b$. Now $(x s a, x s b) \in \rho_{R}$ by Lemma 4 (ii). But

Hence $(z a, z b) \in \rho_{R}$.

$$
x s b=x s a y=z a y=z b .
$$

Let $H$ be a non-zero $\mathscr{H}$-class of $S$. We define a relation $\rho_{H}$ on $H$ by the rule that

$$
(a, b) \in \rho_{H} \Leftrightarrow(s a, s b) \in \rho_{R}, \quad(a, b \in H)
$$

where $s$ is any element of $S^{1}$ such that $s a \in R_{e} \cap L_{a}$. Clearly $\rho_{H}$ is reflexive and symmetric. To see that it is transitive, let $(a, b) \in \rho_{H}$ and $(b, c) \in \rho_{H}$. Then there exists $s \in S^{1}$ such that $s a \in R_{\mathrm{e}} \cap L_{a}$ and $(s a, s b) \in \rho_{R}$. Since $s b \in R_{e} \cap L_{a}$ it follows that $(s b, s c) \in \rho_{R}$ and so $(s a, s c) \in \rho_{R}$ since $\rho_{R}$ is transitive. Thus $\rho_{H}$ is an equivalence on $H$.

The definition in (1) lacks left-right symmetry. We shall now show that we would arrive at the same equivalence on $H$ by using the congruence $\rho_{L}$ on $L_{e}$ defined in the dual form of Lemma 4 (ii).

Again, let $(a, b) \in \mathscr{H}$, where $a \neq 0$ and $b \neq 0$, let $s \in S^{1}$ be such that $s a \in R_{e} \cap L_{a}$ and let $(s a, s b) \in \rho_{R}$. Since $S$ is 0 -bisimple there exists $t \in S^{1}$ such that $a t \in R_{a} \cap L_{e}$. Then $b t \in R_{a} \cap L_{e}$. It will be sufficient to show that $(a t, b t) \in \rho_{L}$. By the definition of $\rho_{R}$ there exusts $g \in N$ such that $g s a=s b$. 
Hence $g s a t=s b t$. Now, by Green's lemma, the mapping

$$
x \rightarrow s x\left(x \in R_{a}\right)
$$

is an $\mathscr{L}$-class-preserving bijection from $R_{a}$ to $R_{\mathrm{e}}$ and so, since at $\in R_{a} \cap L_{e}$, it follows that sat $\in R_{e} \cap L_{e}=H_{e}$. Hence, since $N$ is normal in $H_{e}$, there exists $h \in N$ such that $g($ sat $)=($ sat $) h$. Thus

$$
\text { sath }=s b t .
$$

Now since $(a, s a) \in \mathscr{L}$ there exists $s^{\prime} \in S^{1}$ such that $s^{\prime} s a=a$. But $(a, b) \in \mathscr{H}$ and so $s^{\prime} s b=b$, by Green's lemma. Premultiplying both sides of (2) by $s^{\prime}$ we find that $a t h=b t$. Hence $(a t, b t) \in \rho_{L}$.

Next we define $\rho \subseteq S \times S$ to be $\rho^{*} \cup\{(0,0)\}$, where $\rho^{*}$ is the union of all the subsets $\rho_{H}$ of $S \times S$ as $H$ runs through all the non-zero $\mathscr{H}$-classes of $S$. Since $\rho_{H}$ is an equivalence on $H$ for each $H$, it follows that $\rho$ is an equivalence on $S$. Moreover, $\rho \subseteq \mathscr{H}$. We prove that $\rho$ is a congruence on $S$.

Let $(a, b) \in \rho$ and let $c \in S$. It will be shown that $(c a, c b) \in \rho$. First suppose that $c a=0$. Since $\rho \subseteq \mathscr{H}$ there exists $x \in S^{1}$ such that $a x=b$. Then $c b=c a x=0$. Hence $(c a, c b) \in \rho$. We therefore assume that $c a \neq 0$ and $c b \neq 0$. Since $S$ is 0 -bisimple and $a \neq 0$ there exist $s, s^{\prime} \in S^{1}$ such that $s a \in R_{e} \cap L_{a}$ and $s^{\prime} s a=a$. Then, as before, $s^{\prime} s b=b$. From the definition of $\rho$ we have that $(s a, s b) \in \rho_{R}$. Now esa $=s a$ since $s a \in R_{e}$; therefore

$$
c a=c s^{\prime} s a=c s^{\prime} e s a \text {. }
$$

But $c a \neq 0$. Hence $c s^{\prime} e \neq 0$ and so there exist elements $u, u^{\prime} \in S^{1}$ such that

$$
u c s^{\prime} e \in R_{e} \cap L_{c s^{\prime} e}, u^{\prime} u c s^{\prime} e=c s^{\prime} e \text {. }
$$

Further, $u c s^{\prime} e \in P_{e}$ since $\left(u c s^{\prime} e\right) e=u c s^{\prime} e$. Then, applying Lemma 4 (ii), we find that

$$
\left(u c s^{\prime} e . s a, u c s^{\prime} e . s b\right) \in \rho_{R} \text {. }
$$

But $u c s^{\prime} e s a=u c a$ and $u c s^{\prime} e s b=u c b$; thus

Now

$$
(u c a, u c b) \in \rho_{R} .
$$

$$
u^{\prime} u c a=u^{\prime} u c s^{\prime} e s a=c s^{\prime} e s a=c a
$$

and, similarly, $u^{\prime} u c b=c b$. From (4), (uca, $\left.c a\right) \in \mathscr{L}$ and so, by Green's lemma, the mapping

$$
x \rightarrow u^{\prime} x\left(x \in H_{u c a}\right)
$$

is a bijection from $H_{u c a}$ to $H_{c a}$. Since $\rho_{R} \subseteq \mathscr{H}$ we deduce from (3) that

$$
(c a, c b) \in \mathscr{H} \text {. }
$$

But $u(c a) \in R_{e} \cap L_{c a}$. It then follows from (3) that $(c a, c b) \in \rho$.

In the same way, using the alternative definition of $\rho$ in terms of the equivalence $\rho_{L}$ on $L_{e}$, we can show that $(a c, b c) \in \rho$. Thus $\rho$ is a congruence on $S$.

Finally, let $y \in H_{e}$. Then $(e, y) \in \rho$ if and only if $(s e, s y) \in \rho_{R}$ for any $s \in S^{1}$ such that $s e \in R_{e} \cap L_{e}$. In particular, taking $s=e$, we see that $(e, y) \in \rho$ if and only if $(e, y) \in \rho_{R}$. But $(e, y) \in \rho_{R}$ if and only if $x e=y$ for some $x \in N$. Hence $e \rho=N$. This completes the proof. 
Corollary. Let $S$ be a regular 0-bisimple semigroup and let e be any non-zero idempotent of $S$. Let $H_{e}$ be a left normal divisor of $P_{e}$ and a right normal divisor of $Q_{e}$. Then $\mathscr{H}$ is a congruence on $S$.

Proof. Take $N=H_{e}$ in Lemma 6. Then there exists a congruence $\rho$ on $S$ contained in $\mathscr{H}$ and such that $e \rho=H_{e}$. Then $\rho=\mathscr{H}$ by Lemma 5 (i).

4. Lallement ((2), Theorem 2.3) has shown that the idempotent-separating congruences on a regular semigroup can be characterised as the congruences contained in $\mathscr{H}$. From Lemmas 3,5 and 6 and the corollaries to Lemmas 3 and 6 we then obtain the following theorem concerning the idempotentseparating congruences on a regular 0 -bisimple semigroup.

Theorem. Let $S$ be a regular 0-bisimple semigroup and let e be a non-zero idempotent of $S$. Let $\Lambda$ denote the set of all idempotent-separating congruences on $S$ and let $\Delta$ denote the set of all subgroups of $H_{e}$ that are left normal divisors of $P_{e}$ and right normal divisors of $Q_{e}$. Then

(i) $e \rho \in \Delta$ for all $\rho \in \Lambda$;

(ii) $\rho \subseteq \tau$ if and only if $e \rho \subseteq e \tau(\rho, \tau \in \Lambda)$;

(iii) to each $N$ in $\Delta$ there corresponds $\rho$ in $\Lambda$ such that $e \rho=N$.

Furthermore, $\mathscr{H}$ is a congruence on $S$ if and only if $H_{e} \in \Delta$.

From ((2), Corollary 3.3) we see that $\Lambda$ is a complete modular lattice. The greatest element $\mu$ of $\Lambda$ is the greatest congruence contained in $\mathscr{H}$ and is characterised thus ((4), Lemma 1$)$ :

$$
(a, b) \in \mu \Leftrightarrow(s a t, s b t) \in \mathscr{H} \text { for all } s, t \in S^{\mathbf{1}} .
$$

Let $\Delta$ be partially ordered by inclusion. Then the theorem shows that

$$
\rho \rightarrow e \rho
$$

is an order-preserving bijection from $\Lambda$ to $\Delta$ whose inverse is also orderpreserving. Hence $\Delta$ is a complete modular lattice and $\Delta \cong \Lambda$. A direct calculation establishes that $\Delta$ is a sublattice of the lattice of all normal subgroups of $H_{e}$.

It should also be noted that the theorem provides a description of the idempotent-separating congruences on a regular bisimple semigroup $T$; for $\rho \rightarrow \rho \cup\{(0,0)\}$ is a bijection from the set of all such congruences on $T$ to the set of all idempotent-separating congruences on the regular 0-bisimple semigroup $T^{0}$.

5. We now discuss two important classes of regular 0-bisimple semigroups.

First let $S$ be a completely 0 -simple semigroup. By ((1), Theorem 2.51), $S$ is both regular and 0-bisimple. Let $e$ be a non-zero idempotent of $S$. Then $e$ is primitive and so $S_{e}=H_{e}^{0}\left((\mathbf{1})\right.$, Lemma 2.47). Thus $P_{e}=Q_{e}=H_{e}$. The set $\Delta$ in the theorem therefore consists of all normal subgroups of $H_{e}$ and so there is a natural one-to-one correspondence between the idempotent-separating congruences on $S$ and the normal subgroups of $H_{e}$. In particular, $\mathscr{H}$ is a 
congruence on $S$. These well-known results also follow immediately from the structure theorem for completely 0 -simple semigroups ((1), Theorem 3.5).

Next, let $S$ be a 0 -bisimple inverse semigroup and let $e$ be any non-zero idempotent in $S$. Let $x \in S_{e}$. Then, $x=$ exe and so

$$
x^{-1}=e^{-1} x^{-1} e^{-1}=e x^{-1} e \in S_{e}
$$

This shows that $S_{e}$ is an inverse subsemigroup of $S$. Hence, by Lemma 2, $Q_{e}$ consists of the inverses of the elements of $P_{e}$. Let $N$ be a left normal divisor of $P_{e}$; that is, $a N \subseteq N a$ for all $a \in P_{e}$. Then $N a^{-1} \subseteq a^{-1} N$ for all $a \in P_{e}$ and so $N b \subseteq b N$ for all $b \in Q_{e}$. The set $\Delta$ can therefore be taken as the set of all left normal divisors of $P_{e}$. For a bisimple inverse semigroup the theorem has been given in this form by Reilly and Clifford ((6), Theorem 2.4).

We deduce, in particular, that the idempotent-separating congruences on a bisimple inverse semigroup $S$ with an identity are in one-to-one correspondence with the left normal divisors of the right unit subsemigroup of $S$. This result is due to Warne (7).

6. To conclude, we give an application of the theorem to the principal factors of the full transformation semigroup $\mathscr{T}_{X}$ on a set $X$. It is easy to see that $\mathscr{T}_{\boldsymbol{X}}$ is regular ((1), p. 33, Exercise 1). The further properties required for our discussion-and outlined below-are established in ((1), § 2.2). We remark that Mal'cev (3) has determined a set of generators for the lattice of congruences on $\mathscr{T}_{\boldsymbol{X}}$.

For $\alpha \in \mathscr{T}_{X}$ the equivalence $\alpha_{\circ} \alpha^{-1}$ on $X$ will be denoted by $\pi_{\alpha}$; the cardinal of a set $A$ will be denoted by $|A|$. Then the relations $\mathscr{R}, \mathscr{L}$ and $\mathscr{D}$ on $\mathscr{T}_{\mathrm{X}}$ are characterised as follows:

$$
\begin{aligned}
& (\alpha, \beta) \in \mathscr{R} \Leftrightarrow \pi_{\alpha}=\pi_{\beta}, \\
& (\alpha, \beta) \in \mathscr{L} \Leftrightarrow X \alpha=X \beta, \\
& (\alpha, \beta) \in \mathscr{D} \Leftrightarrow|X \alpha|=|X \beta| .
\end{aligned}
$$

It is also easily verified that if $\alpha, \varepsilon \in \mathscr{T}_{X}$ and $\varepsilon^{2}=\varepsilon$ then

$$
\alpha \varepsilon=\alpha \Leftrightarrow X \alpha \subseteq X \varepsilon \text {. }
$$

Now let $|X|>1$. The principal factors of $\mathscr{T}_{X}$ other than the kernel are of the form $U_{c} / V_{c}$ where $c$ is any cardinal such that $|X| \geqslant c>1$ and $U_{c}, V_{c}$ are the ideals of $\mathscr{T}_{\boldsymbol{X}}$ defined by

$$
U_{c}=\left\{\alpha \in \mathscr{T}_{X}:|X \alpha| \leqq c\right\}, V_{c}=\left\{\alpha \in \mathscr{T}_{X}:|X \alpha|<c\right\}
$$

We write $T_{c}=U_{c} / V_{c}$. Let $\alpha$ be any element of $\mathscr{T}_{X}$ of rank $c$. Then it can readily be shown that, since $\mathscr{T}_{X}$ is regular, the $\mathscr{R}_{\text {-class }} R_{\alpha}$ of $\mathscr{T}_{X}$ is also an $\mathscr{R}$-class of $T_{c}$; similarly, the $\mathscr{L}$-class $L_{\alpha}$ of $\mathscr{T}_{X}$ is an $\mathscr{L}$-class of $T_{c}$. Hence $T_{c}$ is a regular 0-bisimple semigroup. Moreover, by (1), for any non-zero idempotent $\varepsilon$ of $T_{c}$ we have that

$$
P_{\varepsilon}=\left\{\alpha \in T_{c} \mid 0: \pi_{\alpha}=\pi_{\varepsilon} \text { and } X \alpha \subseteq X \varepsilon\right\} .
$$

Consider first the case where $c$ is finite. Let $\varepsilon, \eta$ be non-zero idempotents of 
$T_{c}$ such that $\varepsilon \eta=\eta=\eta \varepsilon$. Since $\eta=\eta \varepsilon$ it follows from (1) that $X \eta \subseteq X \varepsilon$. Thus $X \eta=X \varepsilon$ since $|X \eta|=|X \varepsilon|=c$. Hence $\varepsilon=\varepsilon \eta$ and so $\varepsilon=\eta$. This shows that $T_{c}$ is completely 0 -simple. Since $H_{\varepsilon}$ is isomorphic to the symmetric group of degree $c$, we see that, for $c \geqq 5, T_{c}$ has exactly three distinct idempotentseparating congruences (corresponding to the three distinct normal subgroups of $H_{\mathrm{e}}$ ). Note that one of these congruences is $\mathscr{H}$.

Next let $X$ be infinite and let $c$ be an infinite cardinal such that $|X| \geqq c$. We shall show that the only idempotent-separating congruence on $T_{c}$ is the identity congruence. Let $\varepsilon$ be a non-zero idempotent of $T_{c}$ and let $\gamma$ be an element of $H_{\varepsilon}$ distinct from $\varepsilon$. Then there exists $y \in X$ such that $y \gamma \neq y \varepsilon$. Now $\gamma \in L_{\varepsilon}$ and so $y \gamma \in X \varepsilon$. Since $X \varepsilon$ is infinite there exists an element $\theta$ in $\mathscr{T}_{X}$ that induces a one-to-one mapping of $X \varepsilon$ into $X \varepsilon$ and is such that $y \varepsilon \in(X \varepsilon) \theta$ and $y \gamma \notin(X \varepsilon) \theta$. Write $\alpha=\varepsilon \theta$. Then $\alpha \in T_{c} \mid 0$; also $\pi_{\alpha}=\pi_{\varepsilon}$ and $X \alpha \subseteq X \varepsilon$. Hence, by (2), $\alpha \in P_{\varepsilon}$. But there exists $x \in X$ such that $y \varepsilon=x \alpha$. Therefore, since $\varepsilon \gamma=\gamma$, we have that

$$
x \alpha \gamma=y \varepsilon \gamma=y \gamma \notin X \alpha .
$$

In particular, this shows that $\gamma$ cannot belong to a left normal divisor of $P_{\varepsilon \bullet}$ Hence the only left normal divisor of $P_{\varepsilon}$ is the subgroup $\{\varepsilon\}$ of $H_{\varepsilon}$. It then follows from the theorem that the only idempotent-separating congruence on $T_{c}$ is the identity congruence.

\section{REFERENCES}

(1) A. H. Clifford and G. B. Preston, The Algebraic Theory of Semigroups, vol. 1, Math. Surveys of the American Math. Soc. 7 (Providence, R.I., 1961).

(2) G. Lallement, Congruences et équivalences de Green sur un demi-groupe régulier, C.R. Acad. Sc. Paris, 262 (1966), 613-616.

(3) A. I. Mal'Cev, Symmetric groupoids, Mat. Sbornik, 31 (73) (1952), 136-151.

(4) G. B. Preston, Congruences on completely 0-simple semigroups, Proc. London Math. Soc. (3), 11 (1961), 557-576.

(5) D. REES, On the ideal structure of a semi-group satisfying a cancellation law, Quarterly J. Math. Oxford Ser. 19 (1948), 101-108.

(6) N. R. ReILly and A. H. Clifford, Bisimple inverse semigroups as semigroups of ordered triples (to appear).

(7) R. J. WARNE, The idempotent-separating congruences of a bisimple inverse semigroup with identity (to appear).

\section{UNIVERSITY OF GLASGOW and}

UNIVERSITY OF STIRLING 